\title{
RAPID IDENTIFICATION OF PATHOGENIC STREPTOCOCCI ISOLATED FROM MORIBUND RED TILAPIA (OREOCHROMIS SPP.)
}

\author{
Mohamed ABDElSALAM ${ }^{1}$, Mamdouh Y. ElgEndY ${ }^{2}$, Mohamed SHAALAN ${ }^{3,4 *}$, \\ Mohamed MoustafA ${ }^{1}$ and Masayuki FuJinO ${ }^{5}$ \\ ${ }^{1}$ Department of Fish Diseases and Management, Faculty of Veterinary Medicine, Cairo \\ University, Giza, Egypt; ${ }^{2}$ Department of Hydrobiology, Veterinary Research Division, \\ National Research Centre, Dokki, Giza, Egypt; ${ }^{3}$ Department of Pathology, Faculty of \\ Veterinary Medicine, Cairo University, Giza, Egypt; ${ }^{4} \mathrm{Clinical}$ Division of Fish Medicine, \\ University of Veterinary Medicine, Veterinärplatz 1, A-1210 Vienna, Austria; \\ ${ }^{5}$ National Institute of Infectious Diseases, Tokyo, Japan
}

(Received 14 August 2016; accepted 2 November 2016)

Accurate and rapid identification of bacterial pathogens of fish is essential for the effective treatment and speedy control of infections. Massive mortalities in market-sized red tilapia (Oreochromis spp.) were noticed in mariculture concrete ponds in northern Egypt. Histopathological examination revealed marked congestion in the central vein of the liver with the presence of bacterial aggregates inside the lumen and in the vicinity of the central vein. A total of 12 isolates of streptococci were obtained from the moribund fish. This study documented the ability of the MicroSeq $50016 \mathrm{~S}$ bacterial sequencing method to accurately identify Streptococcus agalactiae and $S$. dysgalactiae mixed infections from moribund red tilapia that were difficult to be recognised by the commercial biochemical systems. The continuously decreasing cost of the sequencing technique should encourage its application in routine diagnostic procedures.

Key words: Streptococcus agalactiae, S. dysgalactiae, red tilapia, MicroSeq 500 , histopathology

Tilapia culture had been practised in Egypt via traditional methods for thousands of years as depicted by paintings on the walls of Egyptian tombs (Bardach et al., 1972). Egypt is now the second largest tilapia producer in the world, after China (Eltholth et al., 2015). The scarcity of freshwater resources and the competition with agriculture and other urban activities oriented the effort towards mariculture (Moustafa et al., 2015). Red tilapia is a promising species for mariculture due to its salinity tolerance (Romana-Eguia and Eguia, 1999). However, the poor spawning performance of red tilapia and its vulnerability to infectious diseases are among the most significant constraints (Hulata et al., 1995).

*Corresponding author; E-mail: Mohamed.Shaalan@vetmeduni.ac.at; Phone: 0043 (1) 250774-733 
Streptococcosis is responsible for considerable losses in global tilapia production (Pretto-Giordano et al., 2010). This disease is caused by a variety of phenotypes belonging to several genera, such as Streptococcus, Lactococcus and Enterococcus. The most severe epizootics are caused by $S$. agalactiae and $S$. iniae (Chen et al., 2007). Recently, $S$. dysgalactiae has also been listed as an emerging fish pathogen (Abdelsalam et al., 2009a,b, 2010a,b, 2013, 2015). All these Streptococcus spp. are also significant zoonotic pathogens.

The identification of these pathogens using commercial biochemical systems has always resulted in misdiagnosis due to the variability of phenotypic traits among given isolates of the same species, and the inconsistency of biochemical results due to the modification in manual procedures adjusted for marine fish pathogens (Verner-Jeffreys et al., 2012). These phenotypic tests might also yield variable results depending on the culture conditions of the bacteria (Hopkins et al., 2001). In addition, 50\% of Streptococcus spp. belonging to Lancefield groups B and C failed to be identified with the Rapid Strep system (Watts and Yancey, 1994). Therefore, comparison of the 16S rRNA sequences of investigated bacteria has high discriminatory power to resolve closely related species (Wagner et al., 2003). MicroSeq500 16S rRNA sequencing is an excellent commercial identification assay marketed for the rapid and accurate identification of bacterial pathogens (Woo et al., 2009) via sequencing the first 500 base pairs of the 16S rRNA gene. The present work was undertaken to investigate the aetiological agents of streptococcal septicaemia in cultured red tilapia in northern Egypt using Microseq 500 assay. The histopathological changes caused by natural streptococcal infections in red tilapia were also investigated in this study.

\section{Materials and methods}

\section{Sampling}

Massive losses in farmed red tilapia (160-250 g) were recorded in concrete ponds in northern Egypt during August 2015. Fish were stocked at densities of $40 \mathrm{fish} / \mathrm{m}^{3}$. Sixty red tilapia and five cohabitant Tilapia zilli (invasive species) were randomly sampled from the investigated farm. Fish specimens were then preserved in isothermal boxes and transferred to the laboratory.

\section{Bacteriological and biochemical examinations}

Loopfuls were obtained from the kidney, liver and brain under completely aseptic condition, then cultured on oxolinic acid-blood agar supplemented with $1.5 \% \mathrm{NaCl}$ and incubated at $25{ }^{\circ} \mathrm{C}$ for $36 \mathrm{~h}$ for the selective isolation of streptococci according to Abdelsalam et al. (2013). Gram staining, haemolysis, oxidase and catalase tests were performed. 


\section{MicroSeq500 16S rDNA sequencing}

Genomic DNA was extracted from the cultivated strains on tryptic soy agar (TSA) (Oxoid) supplemented with $1.5 \% \mathrm{NaCl}$ using prepMan Ultra reagent as described by the manufacturer (Applied Biosystems, USA). A 500-bp to the 5, end segment of the 16S rRNA gene of bacterial strains was amplified using MicroSeq500 16S rDNA Bacterial Identification PCR kit (Applied Biosystems, USA). The PCR products were then purified using a PCR purification kit (Qiagen, Valencia, California). The sequencing reaction consisted of $13 \mu 1$ of the MicroSeq 500 sequencing mix (containing $3.2 \mathrm{pmol}$ of $005 \mathrm{~F}$ and $531 \mathrm{R}$ primers), $4 \mu \mathrm{l}$ of sterile distilled water, and $3 \mu \mathrm{l}$ of purified amplified product. All sequencing analyses were performed in two directions. The sequencing data were analysed and compared with 16S rRNA gene sequences in the GenBank database using the blast program. The nucleotide sequences of the 16S rRNA of Streptococcus sp. strains were submitted to the DNA Data Bank of Japan. The detailed criteria were as follows: identification to the species level was defined as a 16S rRNA gene sequence similarity of $\geq 99 \%$ relative to the prototype strain sequence in GenBank, and identification to the genus level was defined as a 16S rRNA gene sequence similarity of $\geq 97 \%$ relative to the prototype strain sequence in GenBank. Failure to identify the isolate was defined as a 16S rRNA gene sequence similarity score below $97 \%$. The phylogenetic analysis was carried out using MEGA version 5 (Tamura et al., 2011) and compared to related Streptococcus species (S. equisimilis, $S$. iniae and $S$. parauberis). A phylogenetic tree was constructed using the neighbour-joining method with a genetic distance calculated using the Kimura two-step algorithm (substitutions included transitions and transversions, the pattern among lineages assumed homogeneous, and the rate variation among sites uniform) with 1,000 bootstrap replicates.

\section{Histopathological examination}

Specimens from the liver, kidney, spleen and muscles of the infected red tilapia were fixed in $10 \%$ neutral buffered formalin, processed, then embedded in paraffin after dehydration and sectioned at $5 \mu \mathrm{m}$ thickness using a microtome. The sections were stained with haematoxylin and eosin (HE) (Bancroft and Gamble, 2008).

\section{Results}

\section{Clinical examination}

External haemorrhages, skin erosions, opacity and exophthalmia were recorded on the examined fish. Some fish demonstrated a prolapsed and haemorrhagic vent. Internally, the liver, spleen and kidney were congested with the presence of blood-tinged ascitic fluid. The average values measured for dissolved oxygen and un-ionised ammonia were $3 \mathrm{mgl}^{-1}$ and $1.05 \mathrm{mgl}^{-1}$, respectively. 


\section{Identification of bacteria}

Gram-positive cocci arranged in chains were detected. A total number of 9 bacterial isolates were obtained from the samples. The sequencing of bacterial 16S rRNA genes using microseq 500 assay has identified the isolates as $S$. agalactiae (9 isolates) and $S$. dysgalactiae (3 isolates) (Table 1). The blast results of yielded sequences produced $100 \%$ homology with $S$. agalactiae and $S$. dysgalactiae in the GenBank database. Streptococcus agalactiae was detected in water (1 isolate), and also from invading Tilapia zilli ( 1 isolate). The generated phylogenetic tree grouped all $S$. agalactiae isolates in one cluster irrespective of their sources, and they were separated from S. dysgalactiae (Fig. 1). No other bacterial species were detected in any of the investigated samples.

Table 1

Streptococcus spp. isolated in this study

\begin{tabular}{lclll}
\hline No. & Isolate & Source & Streptococcus spp. & $\begin{array}{c}\text { 16S rRNA } \\
\text { Accession no. }\end{array}$ \\
\hline 1 & dys150910 & Red tilapia & S. dysgalactiae & LC093434 \\
2 & dys150911 & Red tilapia & S. dysgalactiae & LC093435 \\
3 & dys150912 & Red tilapia & S. dysgalactiae & LC093436 \\
4 & aga150914 & Red tilapia & S. agalactiae & LC093438 \\
5 & aga150915 & Red tilapia & S. agalactiae & LC093439 \\
6 & aga150916 & Red tilapia & S. agalactiae & LC093440 \\
7 & aga150917 & Red tilapia & S. agalactiae & LC093441 \\
8 & aga150918 & Red tilapia & S. agalactiae & LC093442 \\
9 & aga150919 & Red tilapia & S. agalactiae & LC093443 \\
10 & aga150920 & Red tilapia & S. agalactiae & LC093444 \\
11 & aga150921 & Red tilapia & S. agalactiae & LC093445 \\
12 & aga150922 & Red tilapia & S. agalactiae & LC093446 \\
13 & aga150923 & Tilapia zilli & S. agalactiae & LC093447 \\
14 & aga150926 & Water & S. agalactiae & LC093450
\end{tabular}

\section{Histopathological lesions}

Histopathological examination revealed hyperactivation of the melanomacrophage centres in the liver, kidney and spleen, which was a commonly detected finding. Marked congestion was observed in the central vein of the liver with the presence of bacterial colonies inside the lumen and in the vicinity of the central vein. Additionally, focal necrotic changes and fatty infiltrations of the hepatocytes were also noticed. Mononuclear cell infiltrations were noted between renal tubules. Moreover, infiltration by inflammatory cells between muscle bundles indicated mild myositis. Furthermore, degenerative changes were detected in the muscle bundles (Fig. 2). 


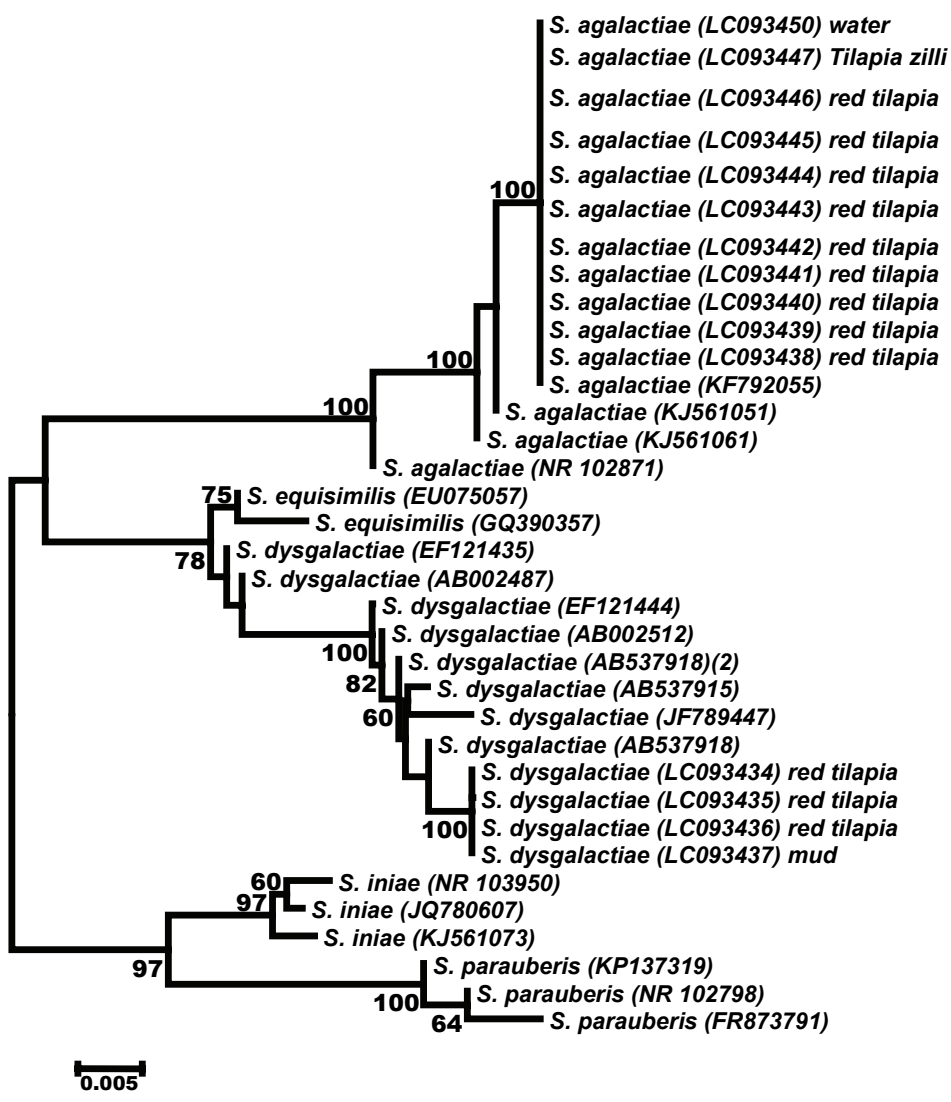

Fig. 1. Phylogenetic tree based on the $16 \mathrm{~S}$ rDNA gene sequences of Streptococcus dysgalactiae and $S$. agalactiae recovered from moribund red tilapia

\section{Discussion}

Streptococcus agalactiae was incriminated in the massive fish kill in the Kuwait bay during 2000-2001 (Evans et al., 2002). It was also isolated from the colossal red tilapia losses in Asia (Abuseliana et al., 2011). Recently $S$. agalactiae has also been incriminated in mass mortalities affecting seabream and seabass cultured in northern Egypt (Elgendy, 2013). Hernandez et al. (2009) recorded mortalities of similar intensity in the brood-stocks of red tilapia. The first epizootic outbreak caused by $S$. dysgalactiae was detected in amberjack (Seriola dumerili) and yellowtail (S. quinqueradiata) in Japan (Nomoto et al., 2004), with necrosis in the caudal peduncle area (Abdelsalam et al., 2013, 2015). The present study documented heavy losses in large-size red tilapia cultured in a marine aquaculture farm in Egypt. The recovered isolates were identified as S. agalactiae and $S$. dysgalactiae. 

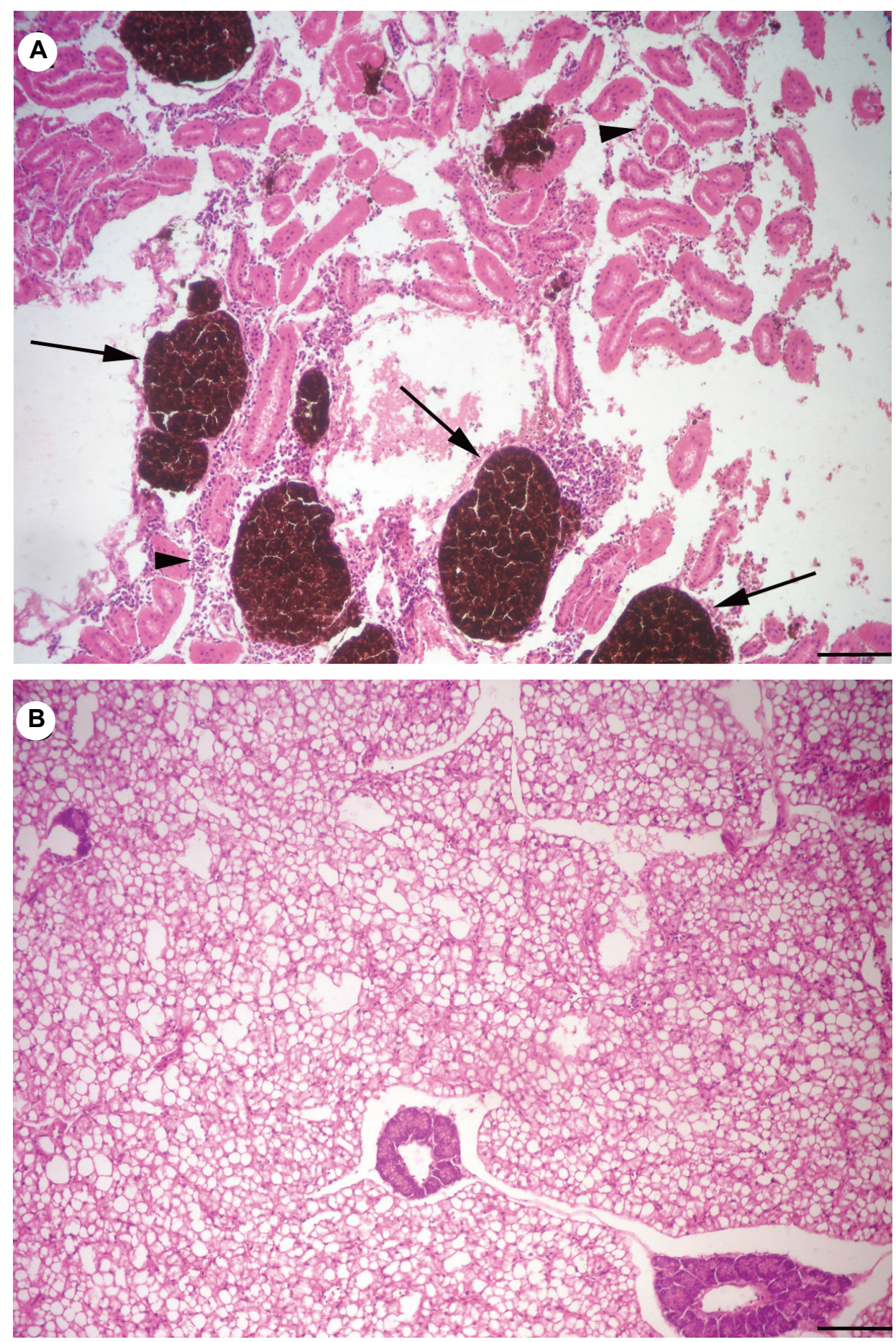


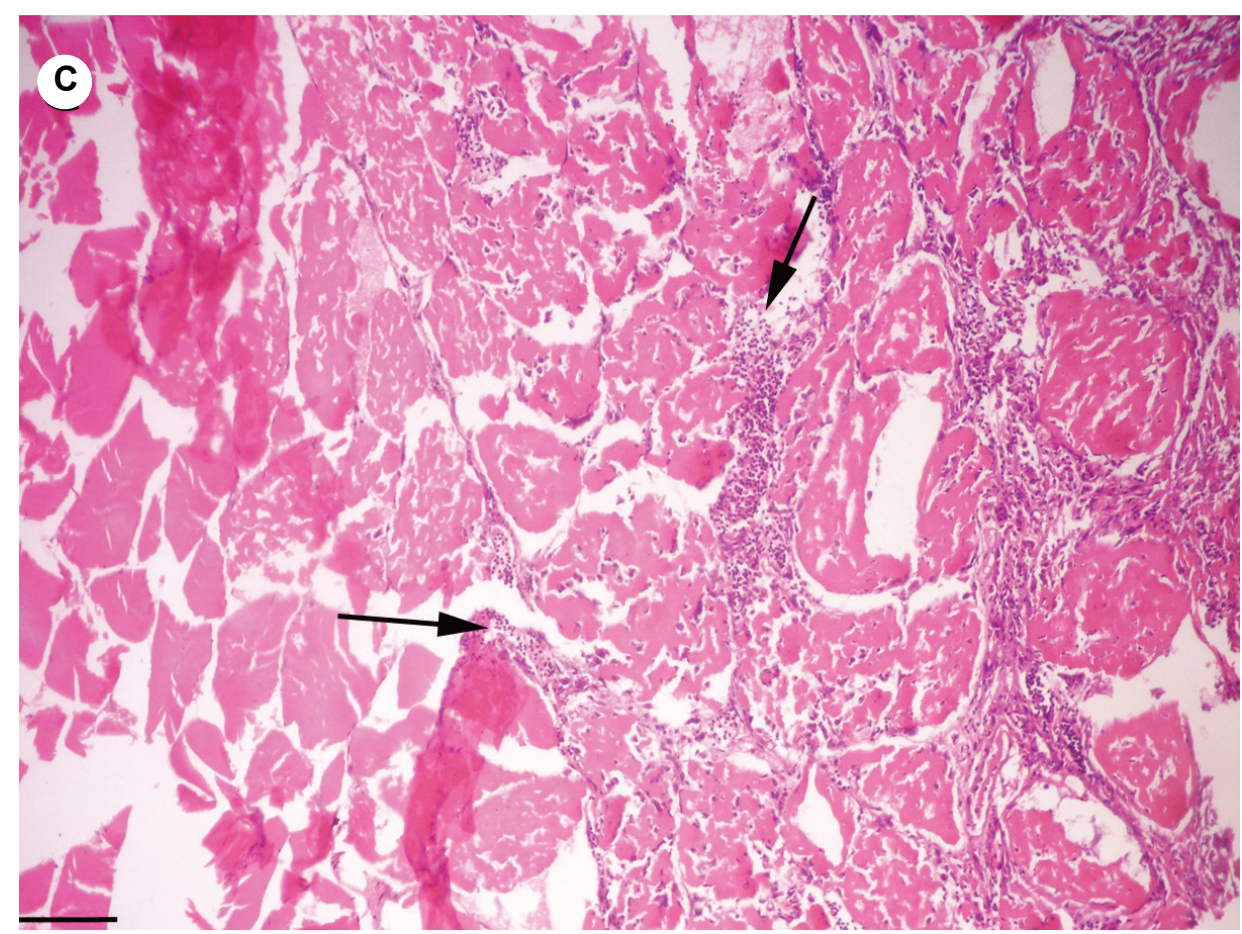

Fig. 2. (A) A microphotograph of the kidney showing mild infiltrations of mononuclear cells between the renal tubules (head arrows) and hyperactivation of the melanomacrophage centres (arrows). Haematoxylin and eosin (HE), scale bar $=50 \mu \mathrm{m}$; (B) A microphotograph of the liver showing fatty infiltrations in the hepatocytes with round regular vacuoles. HE, scale bar $=100 \mu \mathrm{m}$;

(C) A microphotograph of the muscles showing moderate mononuclear cell infiltration between muscle bundles with degenerative changes (arrows). HE, scale bar $=100 \mu \mathrm{m}$

By sequencing the 16S rRNA gene of the retrieved isolates using microseq 500 assay, the identity of the recovered strains was confirmed as $S$. agalactiae and S. dysgalactiae. The blast results of the yielded sequence produced $100 \%$ homology with the $S$. agalactiae and $S$. dysgalactiae GenBank database. In addition, the generated phylogenetic tree confirmed their identities, and $S$. dysgalactiae isolates were well distinguished from those of $S$. agalactiae. This method has proven successful in human and veterinary clinical medicine. Results of the microseq 500 assay proved reliable for the identification and differentiation of Streptococcus spp. in fish. Microbiological analysis indicated the presence of streptococci in water and in $T$. zilli, highlighting the probable source of infections. Overcrowded conditions and high stocking densities are thought to be among the predisposing factors (Abdelsalam et al., 2013). In this study, the mean values recorded for dissolved oxygen and un-ionised ammonia in farming ponds were far from the optimum values. Prolonged exposure to un-ionised ammonia in water with low dissolved oxygen level predisposes tilapias to diseases including streptococcosis (Amal, 2011). 
Various alterations were noticed in the histopathological sections. Hyperactivation of melanomacrophage centres in the haematopoietic organs was frequently detected. The hypertrophy of melanomacrophages is commonly linked with streptococcal infections (Fawzy et al., 2014). Their phagocytic functions as well as sequestration of cellular degradation products have been documented against fish pathogens (Agius and Roberts, 2003). Necrotic and degenerative changes were also detected in various tissues. These alterations are indicative of a systemic infection, which is supported by the isolation of bacteria from various fish tissues. Myositis of the skeletal muscles was observed, and this was also reported in the case of $S$. iniae infection (Lahav et al., 2004). The presence of bacterial aggregations suggestive of streptococci was observed in the histological sections, which was consistent with the results obtained by Zamri-Saad et al. (2010) in red tilapia naturally infected by $S$. agalactiae. This might also indicate the resistance of bacteria to fish defence mechanisms and their role in overwhelming the capacity of the immune system (Chen et al., 2007). The demonstrated histopathological lesions suggested the presence of acute infection and supported the assumption that the haematogenous route is the common way for S. dysgalactiae spread to induce systemic infection (Chang and Plumb, 1996).

\section{Acknowledgement}

The first author gratefully acknowledges the financial support of the Science and Technology Development Fund (STDF), through the Short-term Fellowship program (STDF-STF), under Grant no. 12307.

\section{References}

Abdelsalam, M., Asheg, A. and Eissa, A. E. (2013): Streptococcus dysgalactiae: an emerging pathogen of fishes and mammals. Int. J. Vet. Sci. Med. 1, 1-6.

Abdelsalam, M., Chen, S-C. and Yoshida, T. (2009b): Surface properties of Streptococcus dysgalactiae strains isolated from marine fish. Bull. Eur. Assoc. Fish Pathol. 29, 15-23.

Abdelsalam, M., Chen, S-C. and Yoshida, T. (2010a): Phenotypic and genetic characterization of Streptococcus dysgalactiae strains isolated from fish collected in Japan and other Asian countries. FEMS Microbiol. Lett. 302, 32-38.

Abdelsalam, M., Chen, S-C. and Yoshida, T. (2010b): Dissemination of streptococcal pyrogenic exotoxin G (spegg) with an IS-like element in fish isolates of Streptococcus dysgalactiae. FEMS Microbiol. Lett. 309, 105-113.

Abdelsalam, M., Eissa, A. E. and Chen, S-C. (2015): Genetic diversity of geographically distinct Streptococcus dysgalactiae isolates from fish. J. Adv. Res. 6, 233-238.

Abdelsalam, M., Nakanishi, K., Yonemura, K., Itami, T., Chen, S-C. and Yoshida, T. (2009a): Application of Congo red agar for detection of Streptococcus dysgalactiae isolated from diseased fish. J. Appl. Ichthyol. 25, 442-446.

Abuseliana, A. F., Daud, H. H. M., Aziz, S. A., Bejo, S. K. and Alsaid, M. (2011): Pathogenicity of Streptococcus agalactiae isolated from a fish farm in Selangor to juvenile red tilapia (Oreochromis sp.). J. Anim. Vet. Adv. 10, 914-919. 
Agius, C. and Roberts, R. J. (2003): Melano-macrophage centers and their role in fish pathology. J. Fish Dis. 26, 499-509.

Amal, M. N. A. (2011): Prevalence, risk factors and transmission of Streptococcus agalactiae in the red hybrid tilapia (Oreochromis sp.). PhD Thesis, Faculty of Veterinary Medicine, University Putra Malaysia, Serdang. pp. 161-184.

Bancroft, J. D. and Gamble, M. (2008): Theory and Practice of Histological Techniques. 6th edition. Churchill Livingstone, Edinburgh.

Bardach, J. E., Ryther, J. H. and McLarney, W. O. (1972): Aquaculture: The Farming and Husbandry of Freshwater and Marine Organisms. John Wiley \& Sons Inc., New York. 868 pp.

Chang, P. H. and Plumb, J. A. (1996): Histopathology of experimental Streptococcus sp. infection in tilapia, Oreochromis niloticus, and channel catfish, Ictalurus punctatus. J. Fish Dis. 19, 235-241.

Chen, C., Chao, C. and Bowser, P. R. (2007): Comparative histopathology of Streptococcus iniae and Streptococcus agalactiae-infected tilapia. Bull. Eur. Assoc. Fish Pathol. 27, 2-9.

Elgendy, M. Y. (2013): Epizootiological and molecular studies on the common septicemic bacterial diseases of some saltwater fishes. PhD Thesis, Cairo University, Egypt.

Eltholth, M., Fornace, K., Grace, D., Rushton, J. and Hasler, B. (2015): Characterisation of production, marketing and consumption patterns of farmed tilapia in the Nile Delta of Egypt. Food Policy 51, 131-143.

Evans, J. J., Klesius, P. H., Gilbert, P. M., Shoemaker, C. A., Al Sarawi, M. A., Landsberg, J., Duremdez, R., Al Marzouk, A. and Al Zenki, S. (2002): Characterization of $\beta$-haemolytic Group B Streptococcus agalactiae in cultured seabream, Sparus auratus L., and wild mullet, Liza klunzingeri (Day), in Kuwait. J. Fish Dis. 25, 505-513.

Fawzy, N. M., Osman, K. M., Ibrahim, M., Naguib, M., Ali, M. and Abd-Elrahman, S. (2014): Streptococcosis in tilapia: Clinico-pathological picture of experimentally infected tilapia. Life Sci. J. 11, 1005-1012.

Hernandez, E., Figueroa, J. and Iregui, C. (2009): Streptococcosis on a red tilapia, Oreochromis sp. farm: A case study. J. Fish Dis. 32, 247-252.

Hopkins, M. J., Sharp, R. and Macfarlane, G. T. (2001): Age and disease related changes in intestinal bacterial populations assessed by cell culture, 16S rRNA abundance, and community cellular fatty acid profiles. Gut 48, 198-205.

Hulata, G., Karplus, I. and Harpaz, S. (1995): Evaluation of some red tilapia strains for aquaculture: Growth and color segregation in hybrid progeny. Aquac. Res. 26, 765-771.

Lahav, D., Eyngor, M., Hurvitz, A., Ghittino, C., Lublin, A. and Eldar, A. (2004): Streptococcus iniae type II infections in rainbow trout Oncorhynchus mykiss. Dis. Aquat. Org. 62, 177-180.

Moustafa, M., Eissa, A. E., Laila, A. M., Gaafar, A. Y., Abumourad, I. M. and Elgendy, M. Y. (2015): Investigations into the potential causes of mass kills in mari-cultured gilthead seabream (Sparus aurata) at Northern Egypt. Pharm. Biol. Chem. Sci. 6, 466-477.

Nomoto, R., Munasinghe, L. I., Jin, D. H., Shimahara, Y., Yasuda, H., Nakamura, A., Misawa, N., Itami, T. and Yoshida, T. (2004): Lancefield group C Streptococcus dysgalactiae infection responsible for fish mortalities in Japan. J. Fish Dis. 27, 679-686.

Pretto-Giordano, L. G., Müller, E. E., Klesius, P. and Silva, V. G. D. (2010): Efficacy of an experimentally inactivated Streptococcus agalactiae vaccine in Nile tilapia (Oreochromis niloticus) reared in Brazil. Aquac. Res. 14, 1539-1544.

Romana-Eguia, M. R. and Eguia, R. V. (1999): Growth of five Asian red tilapia strains in saline environments. Aquaculture 173, 161-170.

Tamura, K., Peterson, D., Peterson, N., Stecher, G., Nei, M. and Kumar, S. (2011): MEGA5: Molecular evolutionary genetics analysis using maximum likelihood, evolutionary distance, and maximum parsimony methods. Mol. Biol. Evol. 28, 2731-2739.

Verner-Jeffreys, D., Baker-Austin, C., Pond, M., Rimmer, G., Kerr, R. and Stone, D. (2012): Zoonotic disease pathogens in fish used for pedicure. Emerg. Infect. Dis. 18, 1006-1008. 
Wagner, R., Paine, D. and Cerniglia, C. (2003): Phenotypic and genotypic characterization of competitive exclusion products for use in poultry. J. Appl. Microbiol. 94, 1098-1107.

Watts, J. L. and Yancey, R. J. J. (1994): Identification of veterinary pathogens by use of commercial identification systems and new trends in antimicrobial susceptibility testing of veterinary pathogens. Clin. Microbiol. Rev. 7, 346-356.

Woo, P. C., Teng, J. L., Wu, J. K., Leung, F. P., Tse, H., Fung, A., Lau, S. and Yuen, K. (2009): Guidelines for interpretation of 16S rRNA gene sequence-based results for identification of medically important aerobic Gram-positive bacteria. J. Med. Microbiol. 58, 1030-1036.

Zamri-Saad, M., Amal, M. and Siti-Zahrah, A. (2010): Pathological changes in red tilapias (Oreochromis spp.) naturally infected by Streptococcus agalactiae. J. Comp. Pathol. 143, 227-229. 